Canadian Oncology

Nursing Journal

Revue canadienne

de soins infirmiers

en oncologie

Volume 29, Issue 1 - Winter 2019

elSSN: 2368-8076 


\title{
Population surveillance of navigation frequency and palliative care contact before death among cancer patients
}

\author{
by Grace M. Johnston, Grace Park, Robin Urquhart, Gordon Walsh, Meg McCallum, and Krista Rigby
}

\section{ABSTRACT}

Cancer patient navigation in Canada began in 2002 in Nova Scotia with oncology nurses providing support to patients from diagnosis up to and including end of life. This novel study was carried out to determine navigation frequency and palliative care contact rates, and variations in these rates among adults who were diagnosed with cancer, navigated, and then died between 2011 and 2014. Among the 2,532 study subjects, 56.7\% were navigated for more than one month and $30.6 \%$ had palliative care contact reported. Variations were observed by geographic area, cancer stage, time from diagnosis to death, and whether the person died of cancer. Further study of the role of navigation is advised for persons at end of life.

\section{ABOUT THE AUTHORS}



Grace M. Johnston, PhD, Cancer Registry and Analytics, Nova Scotia Cancer Care Program, Nova Scotia Health Authority, and School of Health Administration, Faculty of Health, Dalhousie University, Halifax, NS

Grace Park, BScN, RN, MD(c), Faculty of Medicine, Dalhousie University, Halifax, NS

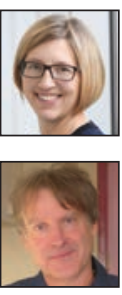

Robin Urquhart, PhD, Faculty of Medicine, Dalhousie University, Halifax, NS

Gordon Walsh, MSc, Nova Scotia Cancer Care Program, Nova Scotia Health Authority, Halifax, NS



Meg McCallum, MA, Nova Scotia Cancer Care Program, Nova Scotia Health Authority, Halifax, NS

Krista Rigby, BScN, RN, MHA, Nova Scotia Cancer Care Program, Nova Scotia Health Authority, Halifax, NS

Corresponding author: Grace M. Johnston, PhD, Professor Emeritus, School of Health Administration, Faculty of Health, Sir Charles Tupper Medical Building, 2nd Floor, Dalhousie University, 5850 College Street, PO Box 15000, Halifax, NS B3H 4R2

Tel: 902-835-9387

Grace.Johnston@dal.ca

DOI:10.5737/236880762911724

\section{INTRODUCTION}

A pproximately $50 \%$ of Canadians will be diagnosed with


Canadians will die of cancer (Canadian Cancer Society [CCS], 2017). Given our aging population, according to Fitch (2017), there will be an increasing number of cancer deaths in the years ahead. Those approaching the end of life are likely to need help navigating the health system. Therefore, this study was carried out to determine navigation frequency and palliative care contact rates, and variations in these rates, among persons diagnosed with cancer who died.

Cancer patient navigators provide education, symptom management, practical and financial support and coordination of care for patients and families across the cancer trajectory from diagnosis to palliative and end-of-life care (Fillion et al., 2012). While cancer patient navigators initially focused on enabling timely screening and diagnosis for vulnerable populations (Paskett, Harrop, \& Wells, 2011; Freeman, \& Rodriguez, 2011), navigation is now being advocated to increase rates of palliative support and advance care planning for persons with a cancer or other advancing life-limiting conditions (Hauser et al., 2011).

Nova Scotia, Quebec (Fillion et al., 2012) and Alberta (Watson, Vimy, Anderson, Champ, \& DeIure, 2016) have published descriptions of their navigation programs, but population-based statistics on cancer navigation are sparse. There have been population-based studies of enrolment in palliative care programs by persons dying of cancer (Gao, Johnston, Lavergne, \& McIntyre, 2011; Craigs, West, Hurlow, Bennett, \& Ziegler, 2018). However, this study and its companion paper (Park, Johnston, Urquhart, Walsh, \& McCallum, 2018) are the first to use a population-based approach to examine the role of the cancer patient navigator in palliative care.

To ensure that all people needing palliative support receive appropriate quality care, population surveillance is essential (CCS, 2010, pp. 70 and 78). The objectives of this study were to identify rates of navigation frequency and palliative care contact, and variations in the rates by demographic, disease and outcome characteristics, among adult cancer patients who were navigated and subsequently died in Nova Scotia. Since rates of access to other forms of cancer palliative care have varied by demographic, disease and outcomes factors (Lavergne, Johnston, Gao, Dummer, \& Rheaume, 2011), we hypothesize that navigation frequency and palliative care contact may vary by demographic, disease and outcome factors. 


\section{METHODS}

\section{Study Setting}

Navigation by experienced oncology nurses in Canada began in Nova Scotia in 2002 (Walkinshaw, 2011; Fillion et al., 2012). Longitudinal data are available for persons approaching the end of their life. Recently, we compared navigated to non-navigated patients who had been diagnosed with cancer (Park, Johnston, Urquhart, Walsh, \& McCallum, 2018). This study builds on that work by investigating only those patients who were navigated to determine navigation frequency and palliative care contact rates.

Multiple patient-related interactions could be made with the navigator in a short period of time. To lessen the time that navigators spend on record submission, navigators submit only one record per month per navigated patient to the provincial cancer program where the information is coded into the cancer registry information system. In this study, we wanted to know whether the navigator had been in communication with or about the person with cancer for a relatively short or a longer period of time.

Issues identified and reported in monthly navigation records are grouped into categories including "coordination" and "contacts/referral". Under each of these two categories, "palliative care" was listed. In addition, "palliative care" was also an option for "referee". For purposes of this study, a check-off in one or more of these three categories on the patient's last navigation record was coded as a "palliative care contact". Since there are specialist palliative care teams across the province, it is assumed that a referral to or from palliative care means the local specialist palliative care team.

\section{Study Design and Data Collection}

A population-based quantitative study was carried out using existing administrative data. The study design was retrospective beginning at the time of death and following study patients back in time through their cancer trajectory to identify navigation, demographic, disease, and outcome information. All data were obtained from the cancer registry information system managed by the Nova Scotia Health Authority. The registry includes all new invasive cancer cases diagnosed since the early 1970s in Nova Scotia, their navigation history, and death certificate information.

\section{Study Population}

The study population included all adults (19 years and over) in Nova Scotia who were diagnosed with invasive cancer, navigated between 2002 and 2014, and died between 2011 and 2014. Decedents who died of cancer were included along with those who were diagnosed with cancer, but had a cause of death that was not cancer. Death certificate-only cases were excluded because their date of diagnosis was their date of death. Central zone was excluded because navigation was not available for all persons diagnosed with cancer in that geographic area. Also excluded were 2011-2012 Eastern zone decedents because navigation was not fully implemented for that zone until 2011.

\section{Variables}

For this study, navigation frequency was defined as the number of monthly navigation records dichotomized into "one" or "more than one" navigation record. Demographic variables (age at death, sex, geographic zone of residence at death, and year of death) were obtained from provincial Vital Statistics death certificates. Three diagnostic variables (cancer diagnosis closest to death, stage at diagnosis, and whether the decedent had prior cancer diagnoses) were obtained from the provincial cancer registry. Whether the decedent died of cancer or not was obtained from their death certificate. Time from last cancer diagnosis to death was created from date of last cancer diagnosis in the cancer registry and date of death from the death certificate. The other outcome variable (hospital as place of death) was obtained from the death certificate.

\section{Data Analysis}

Excel was used to report frequency counts and percentages by decedent characteristics. These statistics were verified using SAS. To protect the confidentiality of the study subjects, counts less than five were not reported. Chi square calculations were carried out using SAS. A p-value of less than 0.05 was used to identify statistically significant variations in rates. Approval for the study was received from the Nova Scotia Health Authority Research Ethics Board.

\section{FINDINGS}

Among the 2,532 study decedents, 1,436 (56.7\%) had more than one navigation record (Table 1). For 776 (30.6\%) of the decedents, a palliative care contact was reported on their last monthly navigation record (Table 2). Almost half $(47.9 \%)$ of the palliative care contacts were a referral by the navigator to a palliative care team (Table 3). In contrast, a small percentage (5.6\%) of navigation records with a palliative care contact were the result of only a referral to the navigator by the palliative care team.

Statistically significant variations in rates were observed for some, but not all demographic factors. Increasing age was significantly associated with fewer monthly navigation records (Table 1). Among persons 19 to 39 years, $72.2 \%$ had multiple navigation records; in contrast, persons 90 years or older had about half that rate (39.0\%). The trend toward increasing age being associated with a decreasing likelihood of a palliative care contact was not statistically significant (Table 2). Sex was not significantly associated with navigation frequency (Table 1) or palliative care contact (Table 2). Geographic region was significantly associated with both navigation frequency and palliative care contact. Year of death was significantly associated with palliative care contact, but not with navigation frequency.

Cancer diagnosis was significantly associated with palliative care contact, but not with navigation frequency. Persons diagnosed with breast cancer had the lowest palliative care contact rates at 19.1\%; the highest rates were for persons diagnosed with pancreas $(42.2 \%)$ and lung $(38.5 \%)$ cancers. Cancer stage at diagnosis was significantly associated with both navigation frequency and palliative care contact. 
While the highest palliative care contact rate was for stage IV (36.2\%), the multiple navigation record rate for stage IV was $56.4 \%$, which was very close to the overall rate of $56.7 \%$. Higher rates of multiple navigation records were seen for stages II (61.5\%) and III (61.9\%). The highest rate (70.5\%) was for the small number $(n=43)$ with stage unknown; they had insufficient diagnostic information on their cancer registry information system to assign a stage.

Having had a prior cancer diagnosis and place of death were not related to either navigation frequency or palliative care contact. In contrast, time from last cancer diagnosis to death and dying of cancer were significantly associated with both navigation frequency and palliative care contact. About a third $(32.8 \%)$ of those who died of cancer had a palliative care contact; fewer $(9.6 \%)$ with a non-cancer death had a palliative care contact.

\section{DISCUSSION}

The findings of this study are rates of navigation frequency and palliative care contact at end of life for persons diagnosed with cancer who were navigated. Among the study patients who died of cancer and were navigated, $32.8 \%$ had a palliative care contact with the cancer patient navigator. Given that only $40 \%$ of the cancer decedents were navigated (Park, Johnston, Urquhart, Walsh, \& McCallum, 2018), this means that only $13.1 \%$ (32.8\% multiplied by 0.4$)$ of the persons dying of cancer had a palliative care contact with a navigator. This rate is low compared to the palliative care program enrolment rates for cancer decedents reported elsewhere. For example, Gao, Johnston, Lavergne, and McIntyre (2011) report a rate of $72 \%$ and Craigs, West, Hurlow, Bennett and Ziegler (2018) report a rate of $65 \%$. In each of these four studies, rates varied significantly by demographic, disease and outcome factors.

In our study, having only one (versus more than one) navigation record increased with age indicating that the navigators were less likely to be involved with older cancer patients over a longer time period. Furthermore, as age increased, patients were also less likely to be navigated (Park, Johnston, Urquhart, Walsh, \& McCallum, 2018), thus placing them in double jeopardy: less likely to be navigated and, when navigated, they were more likely to be navigated for only a short time (a month or less). Duthie, Strohschein and Loiselle (2017) report that as age increases those diagnosed with cancer are more likely to have multi-morbidities that add to the complexity of their care, which influences their treatment options, prognosis, and care needs. Therefore, persons at older ages living with cancer may not be getting the navigation support they need, or their navigation needs are possibly being met by other healthcare providers. Alternatively, persons at younger ages may be getting more navigation than they need, or others on their care team are not adequately meeting the needs of younger persons.

Whether a person was navigated or not varied considerably by cancer diagnosis (Park, Johnston, Urquhart, Walsh, \& McCallum, 2018). However, our study indicated that once persons see a navigator, their navigation frequency did not vary by cancer diagnosis (Table 1), but their palliative care contact rate did not vary by cancer diagnosis (Table 2). Specifically, $30.6 \%$ of the study decedents had a palliative care contact, but this rate varied from about $20 \%$ to $40 \%$ across cancer sites and cancer stage at diagnosis (Table 2). The variations in rates of palliative care contact might be explained by two combining factors: point in cancer trajectory when navigated and survival time.

The lowest rate $(19.1 \%)$ of palliative care contact was for breast cancer. While persons with breast cancer had a higher than average rate of being navigated (Park, Johnston, Urquhart, Walsh, \& McCallum, 2018), our study finding is consistent with the historic focus on cancer screening, early diagnosis, and timely treatment for persons diagnosed with breast cancer (Freeman, 2006; Mertz, et al., 2017). The navigation of breast cancer patients could have occurred years prior to the time of their death, when palliative care was not the focus, since persons with breast cancer have a relatively long survival time (CCS, 2017).

In our study, persons with prostate cancer had an average rate of palliative care contact (27.4\%). This rate was higher than for breast cancer $(19.1 \%)$ even though the survival rates for persons with prostate cancer are longer than for persons diagnosed with breast cancer (CCS, 2017). Our findings might be explained by the fact that persons with prostate cancer had the lowest rate of being navigated likely due to watch and wait often being advisable at diagnosis and urologists not being part of the oncology program (Park, Johnston, Urquhart, Walsh, \& McCallum, 2017). Consequently, when prostate cancer patients were seen by a navigator, this might have been closer to the time of their death. Thus, palliative care may have been more relevant than was the case for the persons with breast cancer who were navigated.

The highest rate of palliative care contact was for persons diagnosed with pancreatic cancer (42.3\%). Their rate of being navigated was lower than average (Park, Johnston, Urquhart, Walsh, \& McCallum, 2017) presumably because a substantial proportion did not receive a medical oncology consultation (Hurton, et al., 2017). Given the relatively short survival time for persons diagnosed with pancreatic cancer (CCS, 2017), it was not surprising that when they were seen by a navigator, almost half of the time there was a palliative care contact.

The findings by cancer stage align with what might be expected given the navigator's cancer treatment enabling role. The rate of multiple navigation records was higher than average for persons diagnosed with stages II and III cancer, and lower for earlier stage cancers. Cancer treatments are typically more aggressive and over a longer period of time for persons diagnosed with stage II and III cancers. While the rate of multiple monthly records was highest for persons with unknown stage at diagnosis, these persons were least likely to be referred to a navigator (Park, Johnston, Urquhart, Walsh, \& McCallum, 2018). The rate of the last navigation being a palliative care contact increased with an increase in stage. This is not unexpected given that the chances of dying from cancer and, thus, the need for palliative care, increases with stage at diagnosis. 


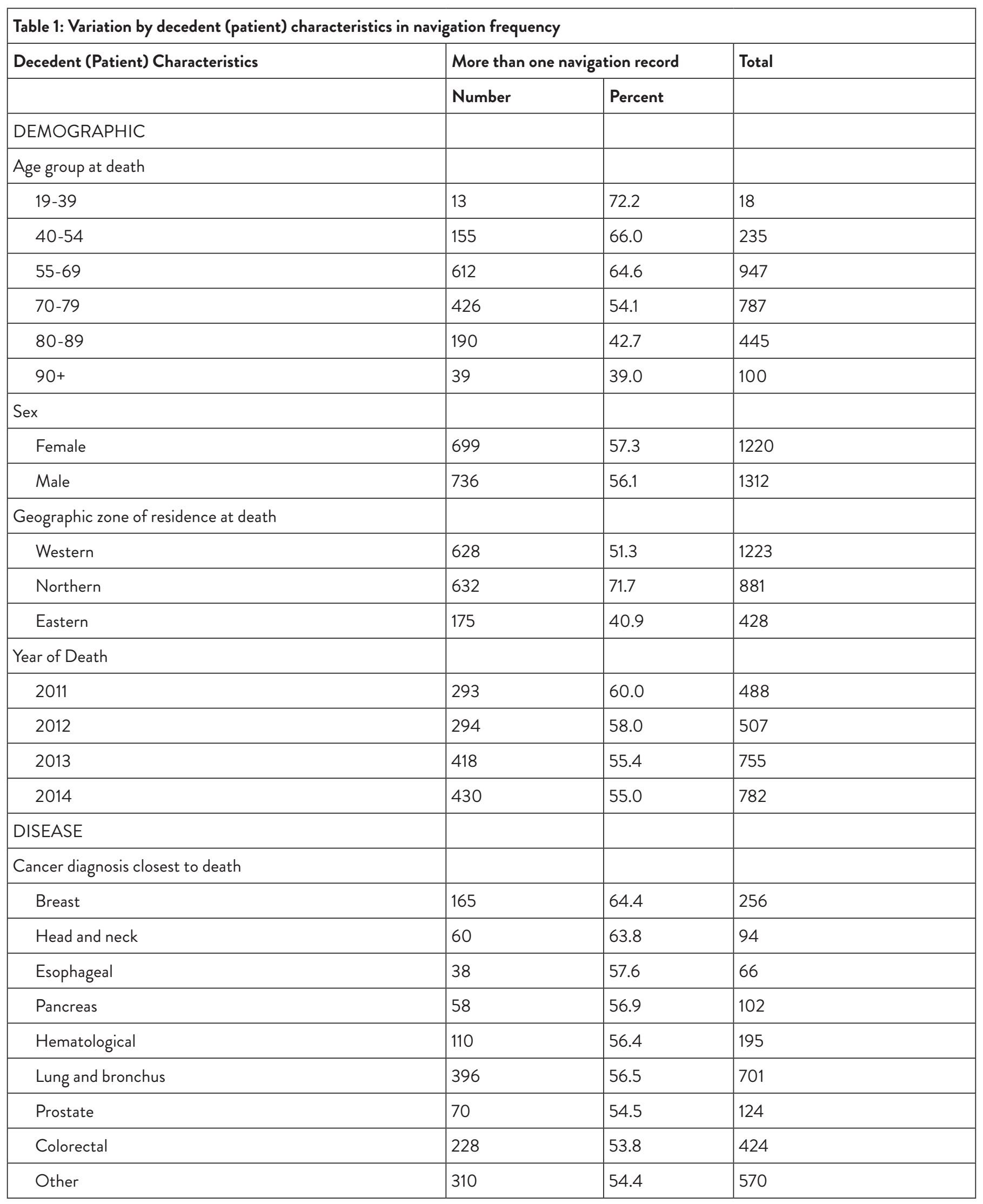




\begin{tabular}{|c|c|c|c|}
\hline \multirow[t]{2}{*}{ Decedent (Patient) Characteristics } & \multicolumn{2}{|c|}{ More than one navigation record } & \multirow[t]{2}{*}{ Total } \\
\hline & Number & Percent & \\
\hline \multicolumn{4}{|l|}{ Cancer stage at diagnosis for last cancer diagnosed ${ }^{a}$} \\
\hline 0 & 10 & 41.7 & 24 \\
\hline I & 139 & 52.1 & 267 \\
\hline II & 190 & 61.5 & 309 \\
\hline III & 284 & 61.9 & 459 \\
\hline IV & 574 & 56.4 & 1018 \\
\hline Unknown ${ }^{b}$ & 43 & 70.5 & 61 \\
\hline Not Available $^{c}$ & 112 & 48.3 & 232 \\
\hline \multicolumn{4}{|l|}{ Prior cancer diagnoses } \\
\hline Yes & 323 & 58.4 & 553 \\
\hline No & 1112 & 56.2 & 1979 \\
\hline \multicolumn{4}{|l|}{ Died of Cancer } \\
\hline Yes & 1338 & 58.1 & 2302 \\
\hline No & 97 & 42.2 & 230 \\
\hline \multicolumn{4}{|l|}{ OUTCOMES } \\
\hline \multicolumn{4}{|l|}{ Time from last cancer diagnosis to death ${ }^{d}$} \\
\hline Less than or equal to 2 weeks & 12 & 25.0 & 48 \\
\hline More than 2 weeks to 13 weeks ( 3 months) & 134 & 36.0 & 372 \\
\hline More than 13 weeks to 26 weeks ( 6 months) & 154 & 53.3 & 289 \\
\hline More than 26 weeks to 52 weeks ( 1 year) & 282 & 64.8 & 435 \\
\hline \multicolumn{4}{|l|}{ Place of Deathe } \\
\hline Hospital & 810 & 55.4 & 1461 \\
\hline Not hospital & 332 & 56.9 & 583 \\
\hline TOTAL & 1435 & 56.7 & 2532 \\
\hline \multicolumn{4}{|c|}{$\begin{array}{l}\text { Findings in bold had a statistically significant association }(p<0.05) \text { between the characteristic and having more than one monthly nav } \\
\text { record, i.e., having multiple records. } \\
\text { a } 162 \text { decedents were omitted because their last cancer diagnosis was before collaborative staging was introduced. } \\
\text { b TNM best stage group could not be determined due to lack of description criteria. } \\
\text { c Cancer could not be staged using TNM Best Stage Group using collaborative stage version } 2.0 \text {. } \\
\text { d More than } 52 \text { weeks omitted because could not ensure all decedents had patient navigator access more than a year prior to death. } \\
\text { e } 488 \text { decedents were omitted since hospital death or not was not available for } 2011 \text { decedents }\end{array}$} \\
\hline
\end{tabular}

Rates of palliative care contacts were not highest for those who were diagnosed within two weeks of death $(25.0 \%)$. Rather, the highest rates were for those diagnosed within three to six months of death (46.7\%) (Table 2). This pattern was consistent with other studies that show that persons who die quickly after a cancer diagnosis were less likely to be referred to a palliative care program (Gao, Johnston, Lavergne, \& McIntyre, 2011). However, since our study showed that navigators were seeing people who died within three months after a cancer diagnosis, there may be opportunities for greater palliative care contact by navigators for patents within three months of death.

This study revealed that while cancer patient navigators were enabling palliative care for about a third $(32.8 \%)$ of the adult cancer survivors who go on to die of cancer, they were also identifying the need for palliative care for about one in 10 $(9.6 \%)$ who die of another condition (Table 2). To date, there has been very little published on the role of cancer navigators in relation to enabling palliative care for cancer survivors facing end of life from non-cancer conditions. 


\begin{tabular}{|c|c|c|c|}
\hline \multicolumn{4}{|l|}{ DEMOGRAPHIC } \\
\hline \multicolumn{4}{|l|}{ Age group at death } \\
\hline $40-54$ & 81 & 34.5 & 235 \\
\hline $55-69$ & 294 & 31.0 & 947 \\
\hline $70-79$ & 246 & 31.3 & 787 \\
\hline \multicolumn{4}{|l|}{ Sex } \\
\hline Female & 354 & 29.0 & 1220 \\
\hline Male & 422 & 32.2 & 1312 \\
\hline \multicolumn{4}{|c|}{ Geographic zone of residence at death } \\
\hline Western & 371 & 30.3 & 1223 \\
\hline Northern & 324 & 36.8 & 881 \\
\hline Eastern & 81 & 18.9 & 428 \\
\hline 2014 & 214 & 27.4 & 782 \\
\hline \multicolumn{4}{|l|}{ DISEASE } \\
\hline \multicolumn{4}{|c|}{ Cancer diagnosis closest to death } \\
\hline Breast & 49 & 19.1 & 256 \\
\hline Colorectal & 92 & 21.7 & 424 \\
\hline Head and neck & 22 & 23.4 & 94 \\
\hline Hematological & 50 & 25.6 & 195 \\
\hline Esophagus & 17 & 25.8 & 66 \\
\hline Prostate & 34 & 27.4 & 124 \\
\hline Lung & 270 & 38.5 & 701 \\
\hline Pancreas & 43 & 42.2 & 102 \\
\hline Other & 199 & 34.9 & 570 \\
\hline
\end{tabular}




\begin{tabular}{|c|c|c|c|}
\hline \multirow[t]{2}{*}{ Decedent characteristics } & \multicolumn{2}{|c|}{ Palliative care contact } & \multirow[t]{2}{*}{ Total } \\
\hline & Number & Percent & \\
\hline \multicolumn{4}{|l|}{ Cancer stage at diagnosis for last cancer diagnosed ${ }^{a}$} \\
\hline$I^{f}$ & 67 & 23.0 & 291 \\
\hline II & 83 & 26.9 & 309 \\
\hline III & 119 & 25.9 & 459 \\
\hline IV & 369 & 36.2 & 1018 \\
\hline Unknown ${ }^{b}$ & 15 & 24.6 & 61 \\
\hline Not Available ${ }^{c}$ & 80 & 34.5 & 232 \\
\hline \multicolumn{4}{|l|}{ Prior cancer diagnoses } \\
\hline Yes & 157 & 28.4 & 553 \\
\hline No & 619 & 31.3 & 1979 \\
\hline \multicolumn{4}{|l|}{ Died of Cancer } \\
\hline Yes & 754 & 32.8 & 2302 \\
\hline No & 22 & 9.6 & 230 \\
\hline \multicolumn{4}{|l|}{ OUTCOMES } \\
\hline \multicolumn{4}{|l|}{ Time from last cancer diagnosis until death ${ }^{d}$} \\
\hline Less than or equal to 2 weeks & 12 & 25.0 & 48 \\
\hline More than 2 weeks to 13 weeks ( 3 months) & 149 & 40.1 & 372 \\
\hline More than 13 weeks to 26 weeks ( 6 months) & 135 & 46.7 & 289 \\
\hline More than 26 weeks to 52 weeks ( 1 year) & 147 & 33.8 & 435 \\
\hline \multicolumn{4}{|l|}{ Place of Deathe } \\
\hline Hospital & 435 & 29.8 & 1461 \\
\hline Not hospital & 166 & 28.5 & 583 \\
\hline TOTAL & 776 & 30.6 & 2532 \\
\hline
\end{tabular}

Table 3: Creation of palliative care contact variable from last navigation record

\begin{tabular}{|l|c|}
\hline Basis for coding Palliative Care Contact & Count (Percent) \\
\hline $\begin{array}{l}\text { Only: Navigator made referral to Palliative } \\
\text { Care }\end{array}$ & $372(47.9)$ \\
\hline $\begin{array}{l}\text { Only: Palliative Care made referral to } \\
\text { Navigator }\end{array}$ & $44(5.6)$ \\
\hline Only: Palliative Care Coordination & $77(9.9)$ \\
\hline $\begin{array}{l}\text { Two of: Palliative Care Referral, Referee, or } \\
\text { Coordination }\end{array}$ & $269(34.7)$ \\
\hline $\begin{array}{l}\text { All of: Palliative Care Referral, Referee, or } \\
\text { Coordination }\end{array}$ & $14(1.8)$ \\
\hline Total & $776(100)$ \\
\hline
\end{tabular}

Strengths, Limitations and Next Steps

Our research makes a substantial contribution to the understanding of palliative care navigation by providing the first population-based epidemiological papers reporting on cancer navigation for persons with cancer approaching the end of their lives. Our studies show that population-based surveillance of navigation is feasible, and can focus on palliative support. While Nova Scotia has population-based data to track trends over time, comparison with other provinces is dependent upon comparable population-based metrics.

More studies are needed to explain findings that might indicate inequities, or might instead reflect how cancer navigation is filling a gap in services that are otherwise unavailable. For example, in this study, having multiple navigation visits varied by geographic zone, and palliative care contact varied by both geographic zone and year. However, it is known that the Eastern zone (which had the lowest palliative care contact rate at $18.9 \%$ ) had a well-established palliative care program associated with its oncology program and so there may be less need 
for navigation involvement in palliative care. In contrast, part of the Northern zone had a shortage of specialized palliative care; the Northern zone had the highest palliative care contact rate $(36.8 \%)$.

With surveillance data in hand, hypotheses can be generated. Do the rates reflect need? What are the reasons for variations in rates? Is the level of palliative care navigator contact adequate for older persons living with cancer? What might be the reason for those who lived only three months or less after their cancer diagnosis having lower palliative care contact rates than those living longer (three to six months) after a cancer diagnosis?

Palliative navigation variables available from our population-based data are limited. Further development of palliative and end-of-life surveillance by navigators can be guided by recommendations for additional outcomes (Hauser, et al., 2011; Fiscella, et al., 2011; Watson, Vimy, Anderson, Champ, \& Delure, 2016). Data quality and interpretation checks are also advised, for example, for persons who died from a non-cancer cause who were seen by a navigator for a palliative reason. Adjustments for associations between variables should also be incorporated. In the future, surveillance reporting could be used to evaluate the extent to which navigation is improving outcomes of interest (Paskett, Harrop, \& Wells, 2011).

\section{REFERENCES}

Canadian Cancer Society's (CCS) Advisory Committee on Cancer Statistics. (2010). Canadian Cancer Statistics 2010. Toronto, ON: Canadian Cancer Society. Retrieved from http://www.cancer.ca/ / media/cancer.ca/CW/cancer\%20information/cancer\%20101/ Canadian\%20cancer\%20statistics/Canadian-Cancer-Statistics2010-EN.pdf?la=en

Canadian Cancer Society's (CCS) Advisory Committee on Cancer Statistics. (2017). Canadian Cancer Statistics 2017. Toronto, ON: Canadian Cancer Society. Retrieved from http://www.cancer.ca/ / media/cancer.ca/CW/cancer\%20information/cancer\%20101/ Canadian\%20cancer\%20statistics/Canadian-Cancer-Statistics2017-EN.pdf?la=en

Craigs, C.L., West, R.L., Hurlow, A., Bennett, M.I., \& Ziegler L.E. (2018) Access to hospital and community palliative care for patients with advanced cancer: A longitudinal population analysis. PLoS ONE 13:8, e0200071.

Duthie, K., Strohschein, F.J., \& Loiselle, C.G. (2017). Living with cancer and other chronic conditions: Patients' perceptions of their healthcare experience. Canadian Oncology Nursing Journal, 27(1), 43-48.

Fillion, L., Cook, S., Veillette, A.M., deSerres, M., Aubin, M., Rainville, F., ... Doll, R. (2012). Professional navigation: A comparative study of two Canadian models. Canadian Oncology Nursing Journal, 22(4), 257-277.

Fiscella, K., Ransom, S., Jean-Pierre, P., Cella D., Stein, K., Bauer, J.E., ... Walsh, K. (2011). Patient-reported outcome measures suitable to assessment of patient navigation. Cancer, 117(15 Suppl.), 3603-3617.

Fitch, M. (2017). Focusing on care of older adults with cancer. Canadian Oncology Nursing Journal, 27(3), 208.

Freeman, H. (2006). Patient navigation: A community based strategy to reduce cancer disparities. Journal of Urban Health, 83(2), 139-141.

Freeman, H.P., \& Rodriguez, R.L. (2011). History and principles of patient navigation. Cancer, 117(15 Suppl.), 3539-3542.

\section{CONCLUSION}

While other population-based studies of access to palliative care have been published, this paper is the first such study of palliative care contact rates for persons at end of life who have accessed cancer patient navigation. This study flags the importance of investigating the rates of cancer-patient navigation and palliative care contact for persons diagnosed with cancer who are at the end of life. Our population-based cancer navigation at end-of-life studies provide a starting point for further examination of the role of cancer navigators in enabling palliative support for persons living with cancer. Population-based surveillance can provide insight into who is being navigated, over what time period and for what reason

\section{FUNDING}

Grace Park was funded by the Dalhousie Medical Research Foundation Chase Summer Studentship. In-kind support was provided by the Nova Scotia Cancer Care Program of the Nova Scotia Health Authority.

Gao, J., Johnston, G.M., Lavergne, M.R., \& McIntyre, P. (2011). Identifying population groups with low palliative care program enrolment using classification and regression tree analysis. Journal of Palliative Care, 27(2), 98-106.

Hauser, J., Sileo, M., Araneta, N., Kirk, R. Martinez, J., Finn, K., ... Rodrigue M.K. (2011). Navigation and palliative care. Cancer, 117(15 Suppl.), 3585-3591.

Hurton, S., Urquhart, R., Kendell, C., Jorgensen, M., Porter, G., Levy, A., \& Moilinary, M. (2017). Variations in medical oncology utilization for pancreatic cancer patients in Nova Scotia. Journal of the Pancreas, 18(10), 62-68.

Lavergne, M.R., Johnston, G.M., Gao, J., Dummer, T.J.B., \& Rheaume, D.E. (2011). Variation in the use of palliative radiotherapy at end of life: Examining demographic, clinical, health service, and geographic factors in a population-based study. Palliative Medicine, 25(2), 101-110.

Mertz, B.G., Dunn-Henriksen, A.K., Kroman, N., Johansen, C., Andersen, K.G., Andersson, M., ... Envold Bidstrup, P. (2017). The effects of individually tailored nurse navigation for patients with newly diagnosed breast cancer: A randomized pilot study. Acta Oncologica, 56(12), 1682-1689.

Park, G., Johnston, G., Urquhart, R., Walsh G., \& McCallum, M. (2018). Comparing enrolees to non-enrolees of cancer patient navigation at end of life. Current Oncology, 25(3),e184-e192.

Paskett, E.D., Harrop, J.P., \& Wells, K.J. (2011). Patient navigation: An update on the state of the science. CA Cancer Journal for Clinicians, 61(4), 237-249.

SAS software, version 9.4. Copyright (C) 2002-2012 SAS Institute Inc. Cary, NC, USA.

Walkinshaw, E. (2011). Patient navigators becoming the norm in Canada. Canadian Medical Association Journal, 183(15), e1109-e1110.

Watson, L.C., Vimy, K., Anderson, J., Champ, S., \& DeIure, A. (2016). Developing a provincial cancer patient navigation program utilizing a quality improvement approach Part three: Evaluation and outcomes. Canadian Oncology Nursing Journal, 26(4), 276-284. 